\title{
Probabilistic Metric Spaces and Hysteresis Systems
}

\author{
T. ERBER
}

Department of Physics, Illinois Institute of Technology, Chicago

B. SCHWEIZER

Department of Mathematics and Statistics, University of Massachusetts, Amherst

\section{A. SKLAR}

Department of Mathematics, Illinois Institute of Technology, Chicago

\author{
Received July 6, 1970
}

\begin{abstract}
A phenomenological theory of simple hysteresis is constructed with the aid of certain concepts from the theory of probabilistic metric spaces. The predicted forms of the dependence of average energy loss per hysteresis cycle on the maximum excursion of the hysteresis coordinate agree well with experimental results.
\end{abstract}

\section{Introduction}

During the last half-century numerous attempts have been made to devise appropriate measures for sets of curves in ordinary or phase spaces. Much of this effort has had its roots in the work of $\mathrm{N}$. Wiener on Brownian motion $[1,2]$ and, in common with Wiener's work, is motivated by problems derived from physics. Indeed, two of the better-known methods are due principally to physicists: these are the methods of lattice enumerations [3], and Feynman path-integrals [4]. In the first, the formidable convergence difficulties that inevitably arise were averted by replacing continuous paths by finite sequences of lattice points; in the second, convergence problems were essentially brushed aside, and results obtained formally under the guidance of physical intuition.

Many of these convergence and general measure-theoretic difficulties arise from the necessity of taking into account paths whose behavior may be indecorous in various ways: nonrectifiable paths; paths that backtrack and retrace themselves, perhaps infinitely often; self-intersecting paths; paths without tangents; etc. Such paths do in fact correspond to current idealizations of the behavior of physical systems in certain situations, e.g., Brownian motion, Zitterbewegung, and turbulence. However, there are non-trivial physical systems in which such mathematical intricacies are ruled out. Specifically, in systems undergoing 
hysteresis, the paths of the points representing the systems in appropriate phase spaces will never backtrack, will cross themselves rarely, if at all, and will be piecewise smooth. In such cases we can on physical grounds approximate each admissible path by a rectifiable arc. A set of such arcs will fill out some region in the enveloping space, and it seems entirely reasonable to adopt the measure of this region as a crude but serviceable measure for the set of arcs. In fact we will show in Section 4 that the quantitative conclusions which are implicit in this choice of measure are in general agreement with experimental results from a wide variety of hysteresis systems.

Specifically, we shall construct a theory which is motivated by the following model: In a metric space with a suitable measure, consider a "test particle" exploring various paths, each a rectifiable arc, from a point $p$ to another point $q$. For any real number $x$, the set of paths from $p$ to $q$ of length not greater than $x$ will cover a certain region, say $R$, of the metric space. Assuming $R$ to be measurable, we then consider the ratio of the measure of $R$ to the measure of some fixed set - perhaps the set covered by all rectifiable arcs joining $p$ to $q$ - to be the probability that the distance between $p$ and $q$ is not greater than $x$ for the test particle.

In this way, we are led to define a particular kind of probabilistic metric space $[5,6]$ which we call an ellipse m-metric. The theory of these spaces is developed in Sections 2 and 3. The specific connection with hysteresis systems is discussed in Section 4, and illustrated by a number of cases of practical interest.

\section{Preliminaries}

For the purpose of facilitating comparison with the later definition of probabilistic metric space we begin with:

Definition 1. A metric space is a pair $(M, d)$, where $M$ is a set whose elements are the points of the space, and the distance functiond is a mapping from $M \times M$ into $R^{+}$(the set of non-negative real numbers) satisfying:

$$
\begin{aligned}
& d(p, p)=0 \quad \text { for all } p \text { in } M ; \\
& d(p, q)>0 \quad \text { for all } p, q \text { in } M \text { if } p \neq q \text {; } \\
& d(p, q)=d(q, p) \quad \text { for all } p, q \text { in } M \text {; } \\
& d(p, r) \leqq d(p, q)+d(q, r) \text { for all } p, q, r \text { in } M .
\end{aligned}
$$

In any metric space the notions of arc, rectifiable arc, and length of a (rectifiable) arc are well-defined (for details, see [7], p. 59). A segment joining points $p, q$ of a metric space is a rectifiable arc with endpoints $p, q$ whose length is exactly $d(p, q)$. Segments, when they exist (they may not!) need not be unique. 
Definition 2. Let $p, q$ be two points of a metric space with distance function $d$, and let $x$ be any real number. The (closed) metric ellipse $E(p, q ; x)$ with foci $p, q$ and major axis $x$ is the set of points $r$ of the metric space such that

$$
d(p, r)+d(r, q) \leqq x .
$$

Clearly, $E(p, q ; x)$ is empty if $x<d(p, q)$, while if $x \geqq d(p, q)$ then $E(p, q ; x)$ contains at least the points $p$ and $q$. In the Euclidean plane, metric ellipses coincide with ordinary ellipses, in Euclidean 3-space with prolate spheroids. If we metrize a 2 -sphere by the usual great-circle distance, then certain metric ellipses stand out by virtue of their simplicity. In particular, if $p$ and $q$ are distinct non-antipodal points on a sphere of radius $\varrho$, then $E(p, q ; \pi \varrho)$ is a hemisphere whose pole is the midpoint of the segment (great circular arc) joining $p$ and $q$, while $E(p, q ; 2 \pi \varrho-d(p, q))$ is the entire sphere. If $p$ and $q$ are antipodal points, then the only nonempty metric ellipse with foci $p, q$ is the entire sphere.

Lemma 1. Let $(M, d)$ be a metric space admitting segments joining any two of its points. For $p, q$ in $M$ and any real number $x$, let $A(p, q ; x)$ denote the set of all points $r$ in $M$ such that $r$ lies on some arc with endpoints $p, q$ whose length does not exceed $x$. Then

$$
A(p, q ; x)=E(p, q ; x) .
$$

Proof. If $x<d(p, q)$, then $A(p, q ; x)$ is empty, since no arc with endpoints $p, q$ can have a length less than $d(p, q)$. Hence (2.6) holds trivially in this case. If $x \geqq d(p, q)$, then both $E(p, q ; x)$ and $A(p, q ; x)$ are nonempty, the latter because it now contains all points on any segment joining $p$ and $q$. Now let $r$ be any point in $A(p, q ; x)$. Then $r$ lies on some arc $\alpha$ with endpoints $p, q$ such that $l(\alpha) \leqq x$, where $l(\alpha)$ is the length of $\alpha$. But by the definition of arc length ([7], p. 59) $l(\alpha) \geqq d(p, r)+d(r, q)$. Hence (2.5) holds, so $r$ is in $E(p, q ; x)$. Conversely, if $r$ is in $E(p, q ; x)$, let $s(p, r)$ denote a segment joining $p$ and $r$, and $s(r, q)$ a segment joining $r$ and $q$. Then $s(p, r)$ and $s(r, q)$ together form a (polygonal) arc with endpoints $p, q$ whose length is

$$
l(s(p, r))+l(s(r, q))=d(p, r)+d(r, q) \leqq x .
$$

Hence $r$ is in $A(p, q ; x)$ and the lemma is proved.

Now it is clear that we want metric ellipses to have proper "areas" or "volumes". In this spirit, we make the following:

Definition 3. A metric space $(M, d)$ is admissible if it (a) admits segments joining any two of its points, and (b) admits a measure $\mu$ such that: (i) every metric ellipse is $\mu$-measurable; (ii) for $p, q$ in $M$ and $x>d(p, q)$ we have

$$
0<\mu(E(p, q ; x))<+\infty \text {. }
$$


In a probabilistic metric space the set of non-negative real numbers $R$ of Definition 1 is replaced by a set of probability distribution functions $\Delta^{+}$. We introduce this set via:

Definition 4. $\Delta^{+}$is the set of all real functions $F$ which are defined on the entire real line, are non-decreasing, right-continuous, and satisfy the conditions

$$
F(x)=0 \text { for } x<0 ; \lim _{x \rightarrow+\infty} F(x)=1 .
$$

N.B. Right-continuity could, without loss, be replaced by some other normalizing condition, e.g. left-continuity.

Among the elements of $\Delta^{+}$are the functions $\varepsilon_{a}$ defined, for any $a$ in $R^{+}$, by:

$$
\varepsilon_{a}(x)= \begin{cases}0, & x<a \\ 1, & x \geqq a .\end{cases}
$$

If $F$ is in $\Delta^{+}$, then various "truncations" of $F$ are also in $\Delta^{+}$. In particular, for $F$ in $\Delta^{+}$and $x$ in $R^{+}$, it is useful to define $F^{(x)}$ by:

$$
F^{(x)}(t)= \begin{cases}0 & t<0, \\ F(t)-F(x)+1 & 0 \leqq t \leqq x, \\ 1 & t \geqq x .\end{cases}
$$

Notice that $F^{(x)}$ differs from the usual truncated distributions in that the "mass" removed from the "tail" of the distribution is concentrated at 0 instead of being redistributed over the entire interval $[0, x]$.

By using some basic properties of Stieltjes integrals, in particular integration by parts, we obtain:

Lemma 2. Let $F$ be in $\Delta^{+}$and $x$ in $R^{+}$. Then we have:

$$
\int_{0}^{\infty} t d F^{(x)}(t)=\int_{0}^{x} t d F^{(x)}(t)=\int_{0}^{x} t d F(t)=x F(x)-\int_{0}^{x} F(t) d t .
$$

Definition 5. A probabilistic metric space (briefly, a $P M$-space) is a pair $(M, \mathscr{F})$, where $M$ is a set whose elements are the points of the space, and the probabilistic distance function $\mathscr{F}$ is a mapping from $M \times M$ into $\Delta^{+}$such that, if we denote the function $\mathscr{F}(p, q)$ by $F_{p q}$ for $p, q$ in $M$, then we have:

$$
\begin{aligned}
& \mathscr{F}(p, p)=F_{p p}=\varepsilon_{0} \text { for all } p \text { in } M . \\
& \mathscr{F}(p, q)=F_{p q} \neq \varepsilon_{0} \text { for all } p, q \text { in } M \text { whenever } p \neq q . \\
& \mathscr{F}(p, q)=F_{p q}=F_{q p}=\mathscr{F}(q, p) \text { for all } \mathrm{p}, \mathrm{q} \text { in } M . \\
& \text { If } F_{p q}(x)=F_{q r}(y)=1 \text {, then } F_{p r}(x+y)=1 \\
& \text { for all } p, q, r \text { in } M \text { and all } x, y \text { in } R^{+} .
\end{aligned}
$$


Comparing this with Definition 1, we see that any metric space gives rise to a $P M$-space via

$$
F_{p q}=\varepsilon_{d(p, q)} .
$$

Conversely, if a $P M$-space $(M, \mathscr{F})$ satisfies (2.15), where $d$ is some mapping from $M \times M$ into $R^{+}$, then we readily see that $d$ is a distance function, and hence $(M, d)$ is a metric space. In this way we can regard metric spaces as special cases of $P M$-spaces.

Of the many types of $P M$-spaces that have been studied (a survey is given in [5]), one particularly elementary type will be relevant in this paper. We introduce it via:

Definition 6. Let $(M, d)$ be a metric space, and $G$ any function in $\Delta^{+}$ other than $\varepsilon_{0}$. The simple space over $(M, d)$ generated by $G$ is the $P M$-space $(M, \mathscr{F})$ in which $\mathscr{F}$ is given by

$$
F_{p q}(x)= \begin{cases}\varepsilon_{0}(x) & p=q, \\ G(x / d(p, q)) & p \neq q .\end{cases}
$$

It is shown in [6] that triangle inequalities stronger than (2.14) hold in any simple space. In particular, we have

$$
F_{p r}(x+y) \geqq \operatorname{Min}\left(F_{p q}(x), F_{q r}(y)\right)
$$

for all $x, y$ in $R^{+}$whenever $p$ and $q$ are points of a simple space. Clearly, (2.17) implies (2.14).

\section{Ellipse $m$-Metrics}

Let $(M, d)$ be an admissible metric space (Def. 3) in which the measure $\mu$ is such that $\mu(M)$ exists and is finite. Then one of the first things which suggests itself is to introduce a $P M$-space on $M$ by defining

$$
F_{p q}(x)= \begin{cases}\frac{\mu(E(p, q ; x))}{\mu(M)} & p \neq q, \\ \varepsilon_{0}(x) & p=q .\end{cases}
$$

Of course this procedure is unavailable if $\mu(M)$ is infinite. Furthermore, it may lead to unwanted side effects even when $\mu(M)$ is finite. This is illustrated by the following example: Let $M$ be a sphere of radius $\varrho, d$ the usual great-circle distance, and $\mu$ the standard Lebesgue measure, so that $\mu(M)=4 \pi \varrho^{2}$. Let $p$ be an arbitrary point of $M$ and $\left\{p_{n}\right\}$ a sequence of points of $M$ distinct from $p$ and converging to $p$. Then $E\left(p, p_{n} ; x\right)$ converges to a circular disk of radius $x / 2$ and area $4 \pi \varrho^{2} \sin ^{2}(x / 4 \varrho)$, whence by (3.1) 
we have:

$$
\lim _{p_{n} \rightarrow p} F_{p p_{n}}(x)= \begin{cases}0 & x \leqq 0, \\ \sin ^{2}(x / 4 \varrho) & 0 \leqq x \leqq 2 \pi \varrho, \\ 1 & x \geqq 2 \pi \varrho .\end{cases}
$$

But $F_{p p}=\varepsilon_{0}$, so $\lim _{p_{n} \rightarrow p} F_{p p_{n}} \neq F_{p p}$. In other words, the probabilistic distance function $\mathscr{F}$ is not continuous. This will be the typical situation whenever $\mathscr{F}$ is defined via (3.1).

An alternative procedure, which works whether $\mu(M)$ is finite or infinite, and leads to continuous $\mathscr{F}$ 's, is suggested by the following considerations: Going back to our original picture of a test particle exploring various paths from $p$ to $q$, it seems not unreasonable to suppose that as soon as the path length exceeds some fixed - and perhaps very large - multiple of the underlying distance $d(p, q)$ between $p$ and $q$, then the particle is certain to arrive at $q$ after having started from $p$. More formally, we make the ansatz that there is a number $m>1$ such that for all $p, q$ in $M$ we have $F_{p q}(x)=1$ whenever $x \geqq m d(p, q)$. (This is a mathematical counterpart of the obvious physical requirement that the phase space trajectories of a system going through successive hysteresis cycles are of bounded length.) These considerations lead to:

Definition 7. Let $(M, d)$ be an admissible metric space with measure $\mu$, and let $m>1$ be fixed. Then the ellipse m-metric on $(M, d, \mu)$ is the $P M$ space on $M$ in which $F_{p q}$ is given by

$$
F_{p q}(x)=\left\{\begin{array}{lll}
\frac{\mu(E(p, q ; x))}{\mu(E(p, q ; m d(p, q)))} & x \leqq m d(p, q) \\
1 & x \geqq m d(p, q)
\end{array}\right\} \begin{aligned}
& p \neq q, \\
& \varepsilon_{0}(x)
\end{aligned}
$$

It is easy to verify from (3.2) that $F_{p q} \Rightarrow \varepsilon_{0}$ if $p \rightarrow q$ and that $F_{p q} \Rightarrow \varepsilon_{d(p, q)}$ if we allow $m$ to approach 1 from above. Here $\Rightarrow$ indicates weak convergence, i.e., convergence at all points of continuity of the limit function.

In many admissible metric spaces, the measure of a metric ellipse depends only on the length of the major axis and the distance between the foci, i.e. there exists a 2-place real function $H$ such that

$$
\mu(E(p, q ; x))=H(x, d(p, q)) .
$$

It may further happen that the function $H$ factors in the sense that there exist non-negative one-place functions $g$ and $h$ such that

$$
H(x, d(p, q))=g\left(\frac{x}{d(p, q)}\right) \cdot h(d(p, q)) .
$$


If (3.4) holds, then it is easy to see that $g$ must be nondecreasing, and $g(t)>0$ for $t>1$. We can therefore define a function $G$ in $\Delta^{+}$via:

$$
G(x)= \begin{cases}0 & x<0, \\ g(x) / g(m) & 0 \leqq x \leqq m, \\ 1 & x \geqq m .\end{cases}
$$

Upon combining (3.2), (3.3), (3.4), and (3.5) we then obtain

$$
F_{p q}(x)=G(x / d(p, q))
$$

for all pairs of distinct points $p, q$ in $M$. Thus we have established

Lemma 3. Let $(M, d)$ be an admissible metric space in which (3.3) and (3.4) hold. Then the ellipse m-metric on $(M, d, \mu)$ is a simple space, for which the function $G$ of Definition 6 has the representation given by (3.5).

Of course the Euclidean plane is the prototype of the set of metric spaces to which Lemma 3 applies. Upon taking $\mu$ to be the ordinary Lebesgue measure in the Euclidean plane, we immediately obtain

$$
\begin{aligned}
\mu(E(p, q ; x)) & =\frac{\pi}{4} x\left(x^{2}-d(p, q)^{2}\right)^{\frac{1}{2}} \\
& =\frac{x}{d(p, q)}\left[\left(\frac{x}{d(p, q)}\right)^{2}-1\right]^{\frac{1}{2}} \frac{\pi}{4} d(p, q)^{2}
\end{aligned}
$$

whenever $x \geqq d(p, q)$. Hence (3.3) and (3.4) hold, with $H, g$, and $h$ given explicitly by:

$$
\begin{aligned}
& H(x, y)= \begin{cases}0 & y \geqq 0, x \leqq y, \\
\frac{\pi}{4} x\left(x^{2}-y^{2}\right)^{\frac{1}{2}} & x \geqq y \geqq 0 ;\end{cases} \\
& g(x)= \begin{cases}0 & x \leqq 1, \\
x\left(x^{2}-1\right)^{\frac{1}{2}} & x \geqq 1 ;\end{cases} \\
& h(y)=\frac{\pi}{4} y^{2} \quad y \geqq 0 .
\end{aligned}
$$

By virtue of Lemma 3 we immediately obtain:

Theorem 1. Let $(M, d)$ be the Euclidean plane and $\mu$ planar Lebesgue measure. Then for any $m>1$ the ellipse m-metric on $(M, d, \mu)$ is the simple space over $(M, d)$ generated by the function $G$ given by:

$$
G(x)= \begin{cases}0 & x \leqq 1, \\ \frac{x}{m}\left(\frac{x^{2}-1}{m^{2}-1}\right)^{\frac{1}{2}} & 1 \leqq x \leqq m, \\ 1 & x \geqq m .\end{cases}
$$


Hence the functions $F_{p q}$ have the following form for $p \neq q$ :

$F_{p q}(x)= \begin{cases}0 & x \leqq d(p, q), \\ \frac{x}{m d(p, q)^{2}}\left(\frac{x^{2}-d(p, q)^{2}}{m^{2}-1}\right)^{\frac{1}{2}} & d(p, q) \leqq x \leqq m d(p, q), \\ 1 & x \geqq m d(p, q) .\end{cases}$

Lemma 2 and some straightforward calculation now yield the following:

Corollary. With $F_{p q}$ as given by (3.10), we have, for any $x \geqq 0$ :

$$
\int_{0}^{\infty} t d F_{p q}^{(x)}(t)= \begin{cases}0 & x \leqq d(p, q), \\ \frac{\left(2 x^{2}+d(p, q)^{2}\right)\left(x^{2}-d(p, q)^{2}\right)^{\frac{1}{2}}}{3 d(p, q)^{2} m\left(m^{2}-1\right)^{\frac{1}{2}}} & d(p, q) \leqq x \leqq m d(p, q), \\ \frac{2 m^{2}+1}{3 m} d(p, q) & x \geqq m d(p, q) .\end{cases}
$$

Similar considerations apply in other Euclidean spaces and lead to:

Theorem 2. Let $(M, d)$ be Euclidean $n$-space $(n \geqq 1)$ and $\mu n$-dimensional Lebesgue measure. Then for any $m>1$, the ellipse m-metric on $(M, d, \mu)$ is the simple space over $(M, d)$ generated by $G_{(n)}$, where

$$
G_{(n)}(x)= \begin{cases}0 & x<1, \\ \frac{x}{m}\left(\frac{x^{2}-1}{m^{2}-1}\right)^{\frac{n-1}{2}} & 1 \leqq x \leqq m \\ 1 & x \geqq m .\end{cases}
$$

Thus $G_{(1)}$ has a jump of height $1 / m$ at 1 and is otherwise piecewise linear, while $G_{(3)}$ is given in the interval $[1, m]$ by

$$
G_{(3)}(x)=\frac{x^{3}-x}{m^{3}-m} .
$$

Our final examples are somewhat more complicated. Let $M$ be a coordinatized affine plane with coordinates $\xi, \eta$, and for $1 \leqq \lambda \leqq \infty$, let $d_{\lambda}$ be the Minkowski distance defined by

$$
\begin{aligned}
& d_{\lambda}\left(\left(\xi_{1}, \eta_{1}\right),\left(\xi_{2}, \eta_{2}\right)\right)=\left(\left|\xi_{1}-\xi_{2}\right|^{\lambda}+\left|\eta_{1}-\eta_{2}\right|^{\lambda}\right)^{1 / \lambda}, \quad 1 \leqq \lambda<\infty, \\
& d_{\infty}\left(\left(\xi_{1}, \eta_{1}\right),\left(\xi_{2}, \eta_{2}\right)\right)=\max \left(\left|\xi_{1}-\xi_{2}\right|,\left|\eta_{1}-\eta_{2}\right|\right) .
\end{aligned}
$$

For each value of $\lambda$, there is a "natural" measure $\mu_{\lambda}$ of area (see [8], pp. 9-10). In particular, $d_{2}$ is the usual Euclidean distance and $\mu_{2}$ the standard planar Lebesgue measure, while for any $\lambda$ there exists a proportionality factor $k_{\lambda}$, depending only on $\lambda$, such that $\mu_{\lambda}=k_{\lambda} \cdot \mu_{2}$. 
Hence in any Minkowski plane relative areas are the same as in the Euclidean plane.

In a non-Euclidean Minkowski plane, the shape of a metric ellipse with foci $p, q$ will generally depend on the direction of the segment joining $p$ and $q$. It follows that (3.3) will not hold in general. However (3.3) and (3.4) will hold in a restricted sense, and in particular for all point-pairs $p, q$ that lie on lines parallel to a fixed line.

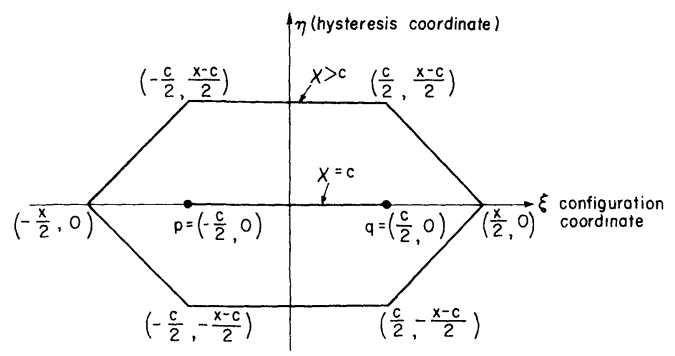

Fig. 1. Metric ellipses in "standard position" in the Minkowski plane with $\lambda=1$

Figure 1 shows metric ellipses in "standard position" for $\lambda=1$, $x \geqq d_{1}(p, q)=c$. The Euclidean area of the ellipse is readily seen to be $\frac{1}{2}\left(x^{2}-d_{1}(p, q)^{2}\right)$. Hence we have

$$
\begin{aligned}
\frac{\mu_{1}(E(p, q ; x))}{\mu_{1}\left(E\left(p, q ; m d_{1}(p, q)\right)\right)} & =\frac{\mu_{2}(E(p, q ; x))}{\mu_{2}\left(E\left(p, q ; m d_{1}(p, q)\right)\right)} \\
& =\frac{x^{2}-d_{1}(p, q)^{2}}{d_{1}(p, q)^{2}\left(m^{2}-1\right)}
\end{aligned}
$$

for $x \geqq d(p, q)$, which yields

Theorem 3. Let $M$ be a coordinatized affine plane, $d_{1}$ the Minkowski distance function defined in (3.13) with $\lambda=1$, and $\mu_{1}$ the corresponding "natural" area measure. Let $p, q$ be distinct points of $M$ such that the line through $p$ and $q$ is parallel to one of the coordinate axes. Then the function $F_{p q}$ in the ellipse m-metric is given by:

$$
F_{p q}(x)=G_{1}\left(x / d_{1}(p, q)\right)
$$

where

$$
G_{1}(x)= \begin{cases}0 & x \leqq 1, \\ \frac{x^{2}-1}{m^{2}-1} & 1 \leqq x \leqq m, \\ 1 & x \geqq m\end{cases}
$$


Again, a direct computation yields the following

Corollary. With $F_{p q}$ as given by (3.14) and (3.15), for any $x \geqq 0$ we have: $\int_{0}^{\infty} t d F_{p q}^{(x)}(t)= \begin{cases}0 & x \leqq d_{1}(p, q), \\ \frac{2}{3} \frac{x^{3}-d_{1}(p, q)^{3}}{d_{1}(p, q)^{2}\left(m^{2}-1\right)} & d_{1}(p, q) \leqq x \leqq m d_{1}(p, q), \\ \frac{2}{3} \frac{m^{2}+m+1}{m+1} d_{1}(p, q) & x \geqq m d_{1}(p, q) .\end{cases}$

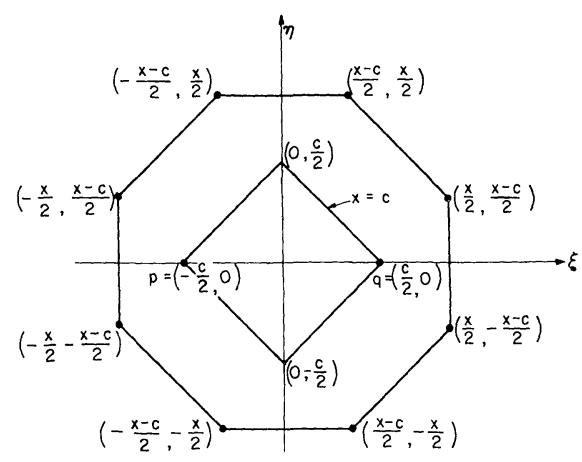

Fig. 2. Metric ellipses in "standard position" in the Minkowski plane with $\lambda=\infty$. Although the figure is symmetric, in physical applications $\eta$ is associated with the hysteresis coordinate and $\xi$ with the configuration coordinate

The other value of $\lambda$ which lends itself to simple illustration is $\lambda=\infty$. Fig. 2 shows two metric ellipses in standard position for the case $\lambda=\infty$, $x \geqq d_{\infty}(p, q)=c$. As in the previous case, simple calculation leads to:

Theorem 4. Let $M$ be a coordinatized affine plane, $d_{\infty}$ the Minkowski distance function defined in (3.13), and $\mu_{\infty}$ the cooresponding area measure. Let $p, q$ be distinct points of $M$ such that the line through $p$ and $q$ is parallel to one of the coordinate axes. Then in the ellipse m-metric on $\left(M, d_{\infty}, \mu_{\infty}\right)$ we have:

$$
F_{p q}(x)=G_{\infty}\left(x / d_{\infty}(p, q)\right)
$$

where

$$
G_{\infty}(x)= \begin{cases}0 & x<1, \\ \frac{2 x^{2}-1}{2 m^{2}-1} & 1 \leqq x \leqq m, \\ 1 & x \geqq m .\end{cases}
$$


Note that $G_{\infty}$ has a jump of height $1 /\left(2 m^{2}-1\right)$ at 1 . This is due to the fact that there are (non-denumerably) many segments joining $p$ and $q$, so the smallest possible non-empty metric ellipse (given by $x=c$ in Fig. 2) already has a non-zero area.

Corollary. With $F_{p q}$ as given by (3.17) and (3.18) and any $x \geqq 0$, we have:

$$
\int_{0}^{\infty} t d F_{p q}^{(x)}(t)= \begin{cases}0 & x<d_{\infty}(p, q), \\ \frac{4 x^{3}-d_{\infty}(p, q)^{3}}{3 d_{\infty}(p, q)^{2}\left(2 m^{2}-1\right)} & d_{\infty}(p, q) \leqq x \leqq m d_{\infty}(p, q), \\ \frac{1}{3} \frac{4 m^{3}-1}{2 m^{2}-1} d_{\infty}(p, q) & x \geqq m d_{\infty}(p, q) .\end{cases}
$$

\section{Applications to Hysteresis Systems}

Many macroscopic physical systems exhibit hysteresis. In the most general terms this means that their response to the variation of some external agency, for instance magnetic fields, stress, etc. is not single valued; or in other words that the evolution of such a system cannot be described in terms of a state function but rather is history-dependent. Such systems were first investigated by Ewing [9]. A general survey of hysteresis is given in [10].

A system undergoing hysteresis will generally dissipate energy, and it is the elucidation of this energy loss that is the principal object of any phenomenological theory of hysteresis. Using the formalism of the previous sections, it is possible to develop such a theory to a quantitative stage with the following procedure:

First we assume that the physical systems can be represented by points in appropriate phase spaces, generally of enormously high dimension. In some cases these high-dimensional "exact" spaces can be replaced by "approximate" phase spaces of much lower dimensionality. In particular, there are systems whose gross behavior can be characterized in a practical sense by a single "phase" parameter: e.g., the magnetization induced by external fields, or the strain manifested in response to external stress. Such systems are the ones we shall consider here. The single remaining "phase" parameter for any such system will be called the configuration coordinate and denoted by $\xi$.

The external agencies which induce the hysteresis will themselves often be specifiable in terms of a finite, though possibly very large, number of parameters. Again, we shall confine our attention to those cases in which the external agencies can be described by means of a single parameter, e.g. "external field strength", "stress", and the like. Such a parameter will be called the hysteresis coordinate and denoted by $\eta$. 
Systems whose behavior can be adequately characterized in terms of the two coordinates $\xi$ and $\eta$ will be called simple hysteresis systems.

Thus a simple hysteresis system (at any given instant) can be visualized as a point in the $(\xi, \eta)$-plane. When $\eta$ is varied, the point will describe some path in the plane; and the existence of hysteresis will be manifested by the fact that if $\eta$ is returned to its initial state, then the corresponding final value of $\xi$ will differ from the original one. A hysteresis cycle will therefore be defined as a path in the $(\xi, \eta)$-plane from a point $p$ to a point $q$ such that $\eta(p)=\eta(q)$, but $\xi(p) \neq \xi(q)$. (Note that this justifies the restrictions in the hypotheses of Theorems 3 and 4.)

Partly on physical grounds (compare [10] and [11]) and partly for the sake of simplicity, we make the following specific assumptions:

1. At each stage of the hysteresis regime of a system there is a distance function $d$ under which the $(\xi, \eta)$-plane becomes a metric space, and which is invariant under translations and reflections in the $\eta$-axis.

2. At each stage the distance between the initial and final point of any hysteresis cycle is fixed. It follows that by translation and reflection we can regard all hysteresis cycles as beginning at a fixed point $p$ and ending at another fixed point $q$.

3. Every hysteresis cycle is a rectifiable arc.

4. The energy loss in a hysteresis cycle is proportional to the length of the cycle for lengths not greater than some fixed multiple $m$ of the distance $d(p, q)[11]$. If the length is greater than this critical value then the energy loss is constant ("saturation").

These assumptions obviously point in the direction of characterizing hysteresis in terms of ellipse $m$-metrics in the $(\xi, \eta)$-plane. We are therefore led to our last assumptions:

5. There is a measure $\mu$ under which the $(\xi, \eta)$-plane with the distance function $d$ is an admissible metric space in the sense of Definition 3.

6. The average energy loss per hysteresis cycle for all hysteresis cycles of length not exceeding $x$ is proportional to

$$
\int_{0}^{\infty} t d F_{p q}^{(x)}(t)
$$

where $F_{p q}^{(x)}$ is the truncation (2.9) of the distance distribution function $F_{p q}$ in the ellipse $m$-metric on the $(\xi, \eta)$-plane (3.2) (cf. $[10,11]$ ).

It should be noted that the number $x$ in (4.1) is not only the maximum permitted path length, but is also, in virtue of Lemma 1, the major axis of a metric ellipse within which all the permitted hysteresis cycles lie. As can be seen from Fig. 3, the major axis of the metric ellipse has the direct physical significance of corresponding to the maximum possible variation in the configuration coordinate $\xi$ over the set of permitted hysteresis cycles. However, from the point of view of practical applica- 
tions, we are also interested in the maximum variation of the hysteresis coordinate $\eta$. According to Fig. 3, this is obviously the minor axis, $y$, of the metric ellipse. If we re-express the integral (4.1) in terms of $y$, the result will evidently depend on the particular distance function $d$, and the particular measure $\mu$. It is easy to obtain explicit results corresponding to the three cases discussed in the preceding section (Theorems 1, 3, 4

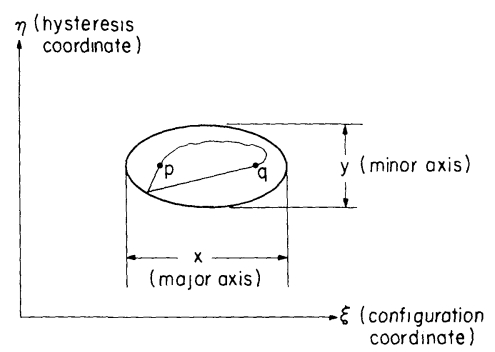

Fig. 3. Metric ellipse in the $(\xi, \eta)$-plane containing hysteresis cycles of length not greater than $x$

and their corollaries). In the Euclidean case (Theorem 1) we obviously have $x=\left(y^{2}+d(p, q)^{2}\right)^{\frac{1}{2}}$, whence the corollary to Theorem 1 yields:

$$
\int_{0}^{\infty} t d F_{p q}^{(x)}(t)= \begin{cases}\frac{2 y^{3}+3 d(p, q)^{2} y}{3 d(p, q)^{2} m\left(m^{2}-1\right)^{\frac{1}{2}}} & y \leqq\left(m^{2}-1\right)^{\frac{1}{2}} d(p, q), \\ \frac{2 m^{2}+1}{3 m} d(p, q) & y \geqq\left(m^{2}-1\right)^{\frac{1}{2}} d(p, q) .\end{cases}
$$

In the Minkowski case with $\lambda=1$, it is clear from Fig. 1 that we have $x=y+d(p, q)$. Then the corollary to Theorem 3 yields:

$\int_{0}^{\infty} t d F_{p q}^{(x)}(t)= \begin{cases}\frac{2}{3} \frac{y^{3}+3 d_{1}(p, q) y^{2}+3 d_{1}(p, q)^{2} y}{d_{1}(p, q)^{2}\left(m^{2}-1\right)} & y \leqq(m-1) d_{1}(p, q), \\ \frac{2}{3} \frac{m^{2}+m+1}{m+1} d_{1}(p, q) & y \geqq(m-1) d_{1}(p, q) .\end{cases}$

Similarly, in the Minkowski case with $\lambda=\infty$, we find from Fig. 2 that $x=y$, and thence from the corollary to Theorem 4 that

$$
\int_{0}^{\infty} t d F_{p q}^{(x)}(t)= \begin{cases}0 & y<d_{\infty}(p, q), \\ \frac{4 y^{3}-d_{\infty}(p, q)^{3}}{3 d_{\infty}(p, q)^{2}\left(2 m^{2}-1\right)} & d_{\infty}(p, q) \leqq y \leqq m d_{\infty}(p, q), \\ \frac{1}{3} \frac{4 m^{3}-1}{2 m^{2}-1} d_{\infty}(p, q) & x \geqq m d_{\infty}(p, q) .\end{cases}
$$


The most striking feature of these expressions is that in all cases they predict a very simple polynomial relation between the average energy loss per hysteresis cycle and the maximum excursion of the hysteresis coordinate. In particular, for $m \gg 1$ the identical cubic dependence is present in all three cases. Clearly the single condition

$$
y \gg d_{(1,2, \infty)}(p, q)
$$

is sufficient to yield the estimate

$$
\int_{0}^{\infty} t d F_{p q}^{(x)}(t) \simeq y^{3},
$$

for all three metric choices. The physical interpretation of (4.5) is that the shift in the state of the system during a hysteresis cycle is small compared to the variation of the hysteresis coordinate. It is well known that this condition most often obtains during asymptotic hysteresis $[10,11]$, and indeed the cubic dependence exhibited by (4.6) then corresponds to the Rayleigh law [ferromagnetic hysteresis] and Dorey's rule [stress-strain hysteresis] which are amply supported by experiment.

Of course it may happen that the shift in the configuration coordinate of a system is comparable to the excursion in the hysteresis coordinate during a cycle. This is likely to occur for virgin hysteresis where the hysteresis loops drift and do not "close", and in the case of shake-down systems where there is a continual drift towards "fatigue" failure [11]. Eqs. (4.2) and (4.3) indicate that in this limit, i.e.

$$
y \sim d_{(1.2)}(p, q)
$$

the ellipse $m$-metrics lead to the linear relation

$$
\int_{0}^{\infty} t d F_{p q}^{(x)}(t) \sim y .
$$

This transition to strict proportionality has also been confirmed experimentally for magnetic cooperative systems and shake-down.

It is remarkable that even the discontinuous Minkowski case (4.4) lends itself to a transparent physical interpretation. In particular, if we consider phase spaces with densely packed instability points and systems that have been cycled to asymptotic hysteresis beyond a second bandedge (see [11]), then the energy losses below this band-edge vanish identically. Eq. (4.4) shows that at the band-edge there is a discontinuous jump $\left(\sim d /\left(2 m^{2}-1\right)\right)$ in the energy losses, and beyond this edge one finds again a cubic dependence. It can be shown that the "shake-down" point of structures is precisely such a discontinuous band-edge, and that the magnitude of the discontinuity is scaled by the Portevin-LeChatelier jumps in the constituent materials. 
Acknowledgments. We are grateful to Professor S. A. Guralnick and Professor H. G. Latal for informative discussions.

This work was supported in part by the Research Corporation and the National Science Foundation.

\section{References}

1. Paley, R. E. A. C., Wiener, N.: Fourier transforms in the complex domain. Am. Math. Soc. Coll. Publ. v. XIX, 1934.

2. Kac, M., et al.: Probability and related topics in physical siences. London, New York: Interscience 1959.

3. Peierls, R.: Ising's model of ferromagnetism. Proc. Cambridge Phil. Soc. 32, 477 (1936).

4. Feynman, R.: The space-time approach to non-relatıvistic quantum mechanics. Rev. Mod. Phys. 20, $367--387$ (1948).

5. Schweizer. B.: Probabilıstic metric spaces -- the first 25 years. New York Statistician 19, no. 2, 3-6 (1967).

6. -, Sklar, A.: Statistical metric spaces. Pacific J. Math. 10. 313-334 (1960).

7. Blumenthal, L. M.: Theory and applications of distance geometry. Oxford: Oxford University Press 1953.

8. Busemann, H.: The geometry of Finsler space. Bull. Am. Math. Soc. 56, 5--16 (1950).

9. Ewing, J. A.: Contributions to the molecular theory of induced magnetism. Proc. Roy. Soc. 48, 342 (1890).

10. Erber, T., Guralnıck, S. A., Latal, H. G.: A general phenomenology for hysteresis (in preparation).

11. - Latal, H. G., Harmon, B. N.: The origin of hysteresis in simple magnetic systems. Advance in Chemical Physics, edited by I. Prigogine and S. Rice. London: Wiley (in press).

T. Erber

Department of Physics

Illinois Institute of Technology

Chicago, Ill. 60616, USA

\section{B. Schweizer}

Department of Mathematics and Statistics Unıversity of Massachusetts, Amherst Mass. 01002, USA
A. Sklar
Department of Mathematics
Illinois Institute of Technology
Chicago, Ill. 60616, USA 